\title{
Phospholipase, proteinase and haemolytic activities of Candida albicans isolated from oral cavities of patients with type 2 diabetes mellitus
}

\section{Correspondence \\ C. S. P. Tsang \\ csptsang@hkucc.hku.hk}

Received 22 March 2007

Accepted 12 June 2007
C. S. P. Tsang, ${ }^{1}$ F. C. S. Chu, ${ }^{1}$ W. K. Leung, ${ }^{1}$ L. J. Jin, ${ }^{1}$ L. P. Samaranayake ${ }^{1}$ and S. C. Siu ${ }^{2}$

\author{
${ }^{1}$ Faculty of Dentistry, The University of Hong Kong, Prince Philip Dental Hospital, Hong Kong SAR, \\ China \\ ${ }^{2}$ Integrated Diabetes Mellitus Research and Training Centre, Department of Medicine and \\ Rehabilitation, Tung Wah Eastern Hospital, Hospital Authority, Hong Kong SAR, China
}

\begin{abstract}
The aim of this study was to biotype and characterize phospholipase, proteinase and haemolytic activities of oral Candida albicans isolates from 210 Chinese patients with type 2 diabetes mellitus (DM) and 210 age- and sex-matched healthy controls. Seventy-six and 50 C. albicans isolates were obtained from type $2 \mathrm{DM}$ patients and controls, respectively, using the oral rinse technique. The isolates were characterized with a biotyping system based on enzyme profiles, carbohydrate assimilation patterns and boric acid resistance of the yeasts, and the isolates were further tested for in vitro phospholipase, proteinase and haemolytic activities. The major biotypes of C. albicans isolates from the type $2 \mathrm{DM}$ and control groups were A1R (42.1\%) and J1R $(36.0 \%)$, respectively. Significantly higher proteinase and haemolytic activities were found in the isolates from the type $2 \mathrm{DM}$ group $(P<0.05)$. Proteinase activity was higher in isolates from patients with $\geqslant 10$ years of DM history than those with $<10$ years $(P<0.05)$. Haemolytic activity was significantly higher in isolates from female DM patients than in those from male counterparts $(P<0.05)$. These data provide evidence of increased extracellular enzyme activity in Candida isolates taken from DM patients.
\end{abstract}

\section{INTRODUCTION}

Oral carriage rates of Candida albicans have been reported to be increased in patients with diabetes mellitus (DM) (Belazi et al., 2005). Extracellular hydrolytic enzymes seem to play an important role in candidal overgrowth (Schaller et al., 2005), as these enzymes facilitate adherence and tissue penetration, and hence invasion of the host. Among the most important hydrolytic enzymes produced by $C$. albicans are phospholipases and secreted aspartyl proteinases (Saps). Furthermore, the ability of C. albicans to acquire elemental iron through haemolysin production is pivotal in its survival and ability to establish infections within humans (Weinberg, 1978).

Seven phospholipase genes (PLA, PLB1, PLB2, PLC1, PLC2, $P L C 3$ and $P L D 1)$ have been identified, but only four of them (PLB1, PLB2, PLC1 and PLD1) have been well characterized (Samaranayake et al., 2006). Their roles in pathogenesis have not been fully elucidated.

Saps are encoded by 10 SAP genes that seem to play different roles in C. albicans infection. SAP1 to SAP6 take

Abbreviations: DM, diabetes mellitus; FPG, fasting plasma glucose; Sap, secreted aspartyl proteinase. part in adherence, tissue damage and evasion of host immune responses. The roles of SAP7 to SAP10 remain elusive, but there is evidence that Sap9 and Sap10 are not secreted from the cell, and instead are regulatory proteinases that play a role in maintaining cell surface integrity (Naglik et al., 2003).

Haemolysin is another putative virulence factor thought to contribute to candidal pathogenesis. In particular, the secretion of haemolysin, followed by iron acquisition, facilitates hyphal invasion in disseminated candidiasis (Odds, 1998).

Few studies have investigated the secretion of both phospholipases and Saps together in C. albicans isolated from DM patients. Furthermore, to our knowledge, the secretion of haemolysin from C. albicans isolated from type 2 DM patients has not been studied before. Therefore, the aims of the present study were (1) to study the biotypes of C. albicans isolated from type $2 \mathrm{DM}$ patients and controls; (2) to determine phospholipase, Sap and haemolysin activities of $C$. albicans isolated from type 2 DM patients; (3) to investigate any correlations between the enzyme profiles of C. albicans isolates and patients' demographic data, smoking habits, denture wearing and DM status; and 
(4) to correlate the C. albicans biotypes with their enzyme profiles.

\section{METHODS}

Subjects. Two hundred and ten Chinese patients aged 41 years or older with type 2 DM were consecutively recruited from the outpatient clinic of the Integrated Diabetes Mellitus Research and Training Centre, Tung Wah Eastern Hospital, Hong Kong SAR, China. Two hundred and ten age- and sex- matched controls without $\mathrm{DM}$, that is, with a fasting plasma glucose (FPG) concentration of $<7.0 \mathrm{mM}$ (Alberti \& Zimmet, 1998), were randomly recruited from the general outpatient clinic of the same hospital during the same period. The controls did not have any systemic disease except essential hypertension.

Data collection. Blood samples were taken from all diabetic patients for FPG and glycosylated haemoglobin $\left(\mathrm{HbA}_{1 \mathrm{c}}\right)$ measurements on the date of study recruitment as part of the patients' regular medical screening or during the baseline DM examination for new cases. A self-administered questionnaire was used to collect demographic data and information on education level, smoking habits and presence of any medical complications. All participants gave written informed consent. Approval for the study was obtained from the Ethics Committee of the Faculty of Dentistry, The University of Hong Kong, Hong Kong SAR, China.

Oral rinse sampling. A modified protocol for concentrated rinse culture was used to evaluate the overall oral yeast carriage (Tsang \& Samaranayake, 2000). The patient was given $10 \mathrm{ml} 0.1 \mathrm{M}$ PBS, $\mathrm{pH} 7.3$, in a sterile universal container and instructed to rinse the mouth for $60 \mathrm{~s}$. Denture-wearing patients did not remove their prostheses. After the oral rinse was expectorated into the container, the sample was transferred immediately to the laboratory, where it was centrifuged at $1700 \mathrm{~g}$ for $10 \mathrm{~min}$. The supernatant was discarded and the pellet resuspended in $2 \mathrm{ml}$ PBS on a vortex mixer for $30 \mathrm{~s}$. A spiral plater (model DU; Spiral Systems) was used to dispense $50 \mu \mathrm{l}$ of the suspension onto various media, as described below.

Culture. The concentrated oral rinse was spiral-plated with an Archimedean spiral onto a Sabouraud glucose agar (SGA) plate (Gibco) and incubated for $48 \mathrm{~h}$ at $37^{\circ} \mathrm{C}$. Well-separated yeast colonies were subcultured onto SGA plates to obtain pure yeast cultures that were then harvested, suspended in water in sterile vials and stored at $-20{ }^{\circ} \mathrm{C}$. The yeast isolates were identified by the germ tube test, growth at $45^{\circ} \mathrm{C}$, chlamydospore formation and API 20C AUX (bioMérieux) assimilation tests. Their phenotypes were further defined by culture on CHROMagar Candida plates. Their identities were reconfirmed with the API AB Plus (bioMérieux) assay to exclude Candida dubliniensis. The yeasts were then stored in vials with multiple glass beads (Microbank) at $-70{ }^{\circ} \mathrm{C}$, subcultured monthly on SGA and maintained at $4{ }^{\circ} \mathrm{C}$ during the experimental period, unless otherwise stated. The purity of the cultures was confirmed periodically by Gram-staining and the germ tube test.

C. albicans biotyping. All yeast isolates were biotyped with two commercially available biotyping kit systems, API ZYM and API 20C AUX (bioMérieux) (Leung et al., 2000), and a boric acid-resistance test (Williamson et al., 1987; Tsang et al., 1995). In brief, the API ZYM system evaluated the enzyme activity of the isolates by means of a battery of 19 enzyme substrates contained in miniaturized plastic cupules of a multi-cupule tray. Colour reactions in each cupule were measured after inoculation of a standard quantity of C. albicans suspension, incubation for $4 \mathrm{~h}$ at $37{ }^{\circ} \mathrm{C}$ and addition of reagents according to the manufacturer's instructions. The API 20C AUX system measured the ability of C. albicans isolates to assimilate 19 different carbohydrates as sole sources of carbon. The results were obtained from a comparison of the extent of opacity in test and control cupules. The boric acid-resistance test assessed the sensitivity of the isolates to $1.8 \mathrm{mg}$ boric acid $\mathrm{ml}^{-1}$ incorporated into the agar medium.

Determination of phospholipase activity. C. albicans isolates were screened for extracellular phospholipase activity by measuring the size of the zone of precipitation after growth on egg yolk agar (Samaranayake et al., 1984). The egg yolk medium consisted of $13.0 \mathrm{~g}$ SGA (Oxoid), $11.7 \mathrm{~g} \mathrm{NaCl}, 0.11 \mathrm{~g} \mathrm{CaCl}_{2}$ and $10 \%$ sterile egg yolk (Oxoid) (all in $184 \mathrm{ml}$ distilled water). First, the components without the egg yolk were mixed and sterilized, then the egg yolk was centrifuged at $500 \mathrm{~g}$ for $10 \mathrm{~min}$ at room temperature and $20 \mathrm{ml}$ of the supernatant was added to the sterilized medium. Standard inocula of the test and control Candida isolates $\left[5 \mu \mathrm{l}\right.$, with $10^{8}$ yeast cells $(\mathrm{ml}$ saline $)^{-1}$ ] were deposited onto the egg yolk agar medium and left to dry at room temperature. A further $5 \mu$ of saline, but without yeast cells, was overlaid onto the plate and left to dry at room temperature. Each culture was then incubated at $37{ }^{\circ} \mathrm{C}$ for $48 \mathrm{~h}$, after which the diameter of the precipitation zone around the colony (an indicator of phospholipase activity) was determined.

The plates were read with the aid of a computerized image analysis system (Quantimet 500 Qwin; Leica), which measured the diameter of the colonies relative to the precipitation zones on a magnified scale. Phospholipase activity $\left(\mathrm{P}_{\mathrm{z}}\right.$ value $)$ was expressed as the ratio of the diameter of the colony to the diameter of the colony plus the precipitation zone (in $\mathrm{mm}$ ) (Price et al., 1982). The assay was conducted in duplicate on three separate occasions for each yeast isolate tested. Reference strains of C. albicans (ATCC 10231 and ATCC 24433) served as positive controls. A strain of Candida glabrata from our laboratory stock collection served as a negative control.

Determination of proteinase activity. Extracellular proteinase activity of C. albicans strains was analysed in terms of BSA degradation according to the technique described by Staib (1965). In brief, an $18 \mathrm{~h}$ yeast suspension of $1 \times 10^{6} \mathrm{cells} \mathrm{ml}^{-1}$ was prepared, and $10 \mu \mathrm{l}$ suspension was inoculated onto a $1 \%$ BSA plate. The plate was incubated for 5 days at $37{ }^{\circ} \mathrm{C}$, flooded with $1.25 \%$ naphthalene black solution for $15 \mathrm{~min}$ and washed with $90 \%(\mathrm{v} / \mathrm{v})$ methanol/water destaining solution, followed by $36 \mathrm{~h}$ of decolourization with several changes of destaining solution.

Results were examined and quantified by a computerized image analysis system (Quantimet 500 Qwin; Leica). The ratio of the diameter of the colony to that of the clear zone of proteolysis (in $\mathrm{mm}$ ) was used as an index $\left(\operatorname{Pr}_{\mathrm{z}}\right.$ value) to represent the extent of proteinase activity by the different $C$. albicans isolates. The assay was conducted in duplicate on three separate occasions for each yeast isolate tested. Reference strains of C. albicans (ATCC 10231 and ATCC 10261) served as positive controls. Candida parapsilosis ATCC 22019 served as a negative control.

Determination of haemolysin activity. Haemolysin activity was evaluated with a blood plate assay (Manns et al., 1994; Luo et al., 2001). Media were prepared by adding $7 \mathrm{ml}$ fresh sheep blood (Dixon) to $100 \mathrm{ml} \mathrm{SGA} \mathrm{(Merck)} \mathrm{supplemented} \mathrm{with} \mathrm{glucose} \mathrm{at} \mathrm{a} \mathrm{final}$ concentration of $3 \%(\mathrm{w} / \mathrm{v})$. The final $\mathrm{pH}($ mean $\pm \mathrm{SD})$ of the medium was $5.6 \pm 0.2$. A standard inoculum of both the test and the control Candida isolates $\left.\left[10 \mu \mathrm{l} \text {, with } 10^{8} \text { yeast cells ( } \mathrm{ml} \text { saline }\right)^{-1}\right]$ was deposited onto the medium. A further $10 \mu \mathrm{l}$ of saline but without yeast cells was overlaid onto the same plate. The plate was then incubated at $37{ }^{\circ} \mathrm{C}$ in $5 \% \mathrm{CO}_{2}$ for $48 \mathrm{~h}$.

After incubation, plates were examined and colonies quantified by a computerized image analysis system (Quantimet 500 Qwin; Leica). 
The ratio of the diameter of the colony to that of the translucent zone of haemolysis (in mm) was used as the haemolytic index $\left(\mathrm{H}_{\mathrm{z}}\right.$ value) to represent the extent of haemolysin activity by different $C$. albicans isolates. The assay was conducted in duplicate on three separate occasions for each yeast isolate tested. A reference strain of C. albicans (ATCC 90028) served as a positive control. In addition, one strain each of Streptococcus pyogenes (Lancefield group A) and Streptococcus sanguis, which induce beta and alpha haemolysis, were used as positive controls. Candida parapsilosis ATCC 22019 served as a negative control.

Data analysis. Statistical analyses were performed using the Student's $t$ test, chi square test, Fisher's exact test and correlation coefficient. A $P$ value of 0.05 was considered significant.

\section{RESULTS}

The mean \pm SD age of the diabetic group was $64 \pm 10$ years; $54 \%(n=113)$ were female. These results were similar for the control group. The two groups were also similar in terms of education (e.g. $51 \%$ of controls and $60 \%$ of DM patients had received no or only primary education) and smoking habits (e.g. $71 \%$ of controls and $72 \%$ of DM patients were non-smokers). Controls and DM patients were significantly different, however, in terms of hypertension ( 43.8 vs $63.3 \%)$, denture wearing ( 34.8 vs $47.1 \%$ ) and $C$. albicans isolation (23.8 vs $36.2 \%$ ) (all $P$ values $<0.05$ ).

The mean age of DM onset was 56 years, and the duration of diabetes was 6.8 and 8.9 years in male and female diabetic patients, respectively. The mean $\mathrm{HbA}_{1 \mathrm{c}}$ was $7.8 \pm$ $1.3 \%$ for male diabetic patients and $8.0 \pm 1.3 \%$ for female patients. The mean FPG was $8.3 \pm 2.4 \mathrm{mM}$ in male patients and $8.6 \pm 2.9 \mathrm{mM}$ in female patients.

The oral carriage of $C$. albicans in the test group $(n=76$; $36.2 \%)$ was significantly greater than that in the control group $(n=50 ; 23.8 \%)$ (chi square test, $P<0.05)$. Other Candida species isolated included Candida tropicalis (five isolates from DM and two from control groups), C. glabrata (five isolates from DM and three from control groups), $C$. parapsilosis (three isolates from DM and two from control groups), Candida guilliermondii (one isolate from the control group) and Candida famata (one isolate from the DM group). Fourteen diabetic patients harboured two Candida species simultaneously, while eight non-diabetic patients harboured two Candida species.

\section{Biotype}

The biotype profiles of the C. albicans are shown in Fig. 1. Altogether, 11 biotypes were detected (six from the test group and eight from the control group). Three biotypes were detected in both groups (A1S, J1R and J1S). The major biotype detected in the test group was A1R $(n=32$; $42.1 \%$ ), whereas J1R was the major biotype detected in the control group $(n=18 ; 36 \%)$. Significantly more C. albicans isolates of API ZYM biotype A than any other were detected in the test group (Fisher's exact test, $P<0.0001$ ), whereas significantly more isolates of API ZYM biotype J than any other were detected in the control group (Fisher's exact test, $P<0.0001)$. No correlations could be found between biotype and age or sex in general, or between testgroup biotypes and $\mathrm{HbA}_{1 \mathrm{c}}$ or FPG.

Of the 126 C. albicans isolates (test and control) tested, all were found to be positive for phospholipase, Sap and haemolysin activities.

\section{Phospholipase}

The $\mathrm{P}_{\mathrm{z}}$ values of the C. albicans isolates ranged from 0.68 to 0.89 for the DM group and from 0.65 to 0.89 for the controls. No significant difference in phospholipase activity could be detected between the isolates from the test and control groups (Table 1). No significant association could

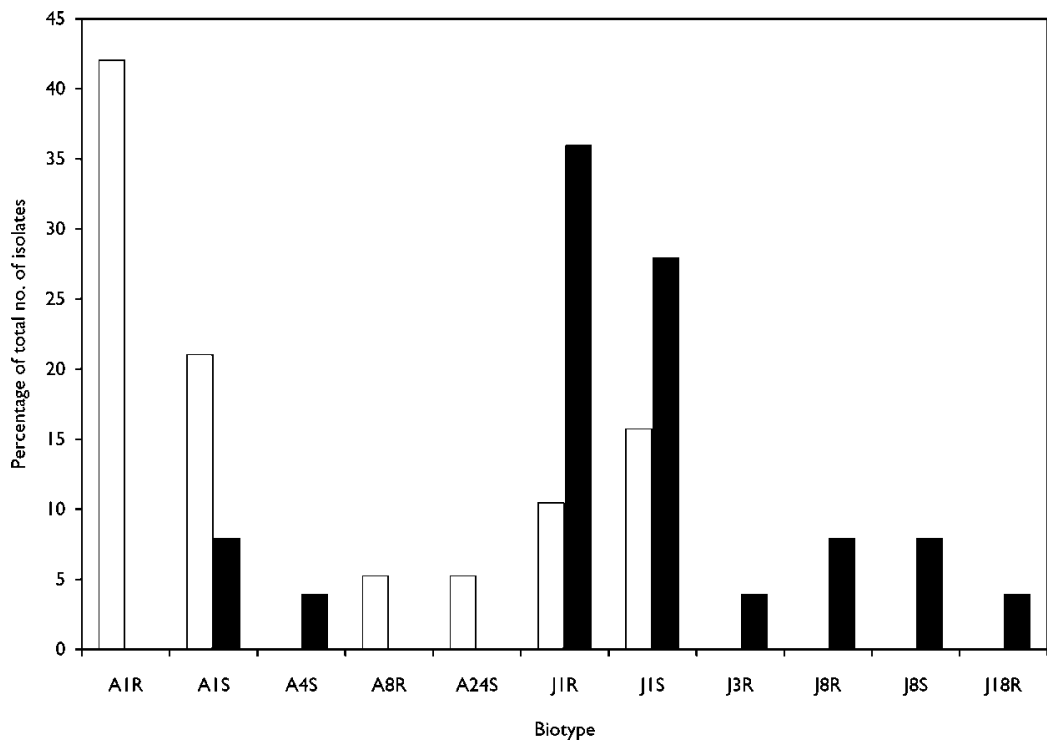

Fig. 1. Biotypes of Candida albicans isolates from diabetic (open bars) and non-diabetic (closed bars) subjects. The $y$ axis shows the number of isolates in each biotype as a percentage of the total number of isolates. 
Table 1. Phospholipase, proteinase and haemolysin activities of oral C. albicans isolates from diabetic and non-diabetic subjects

Results are expressed as mean $\pm \mathrm{SD}$. NS, Not significant.

\begin{tabular}{|lccccc|}
\hline Activity & $\begin{array}{c}\text { Non-diabetic group, } \\
\boldsymbol{n}=\mathbf{5 0}\end{array}$ & $\begin{array}{c}\text { Diabetic group, } \\
\boldsymbol{n}=\mathbf{7 6}\end{array}$ & Test & Statistic & $\boldsymbol{P}$ value \\
\hline Phospholipase $\left(\mathrm{P}_{\mathrm{z}}\right)$ & $0.794 \pm 0.060$ & $0.776 \pm 0.050$ & $t$ & 1.43 & NS \\
Proteinase $\left(\mathrm{Pr}_{\mathrm{z}}\right)$ & $0.517 \pm 0.156$ & $0.425 \pm 0.122$ & $t$ & 3.1 & 0.0027 \\
Haemolysin $\left(\mathrm{H}_{\mathrm{z}}\right)$ & $0.764 \pm 0.08$ & $0.673 \pm 0.06$ & $t$ & 4.71 & $<0.0001$ \\
\hline
\end{tabular}

be detected between $\mathrm{P}_{\mathrm{z}}$ and biotype. Similarly, no significant association was observed between phospholipase activities of $C$. albicans isolates from type $2 \mathrm{DM}$ patients and demographic data, smoking habit, denture-wearing status, $\mathrm{HbA}_{1 \mathrm{c}}$, FPG or duration of diabetes.

\section{Proteinase}

The $\operatorname{Pr}_{z}$ values ranged from 0.23 to 0.79 for the DM group and from 0.30 to 0.87 for the controls. The mean proteinase activity of isolates from the test group $(0.425 \pm 0.122)$ was significantly higher than that of the control isolates $(0.571 \pm 0.156$; Table 1$)$. C. albicans isolated from type 2 DM patients with a diabetes history of $\geqslant 10$ years had a lower mean $\operatorname{Pr}_{z}(0.428 \pm 0.081)$, i.e. higher proteinase activity, than patients with a diabetes history of $<10$ years $(0.548 \pm 0.168)$ (Student's $t$ test, $P<0.05)$. There were no significant associations between $\operatorname{Pr}_{\mathrm{z}}$ and biotype. No correlation could be found between $\mathrm{Pr}_{\mathrm{z}}$ of isolates from type $2 \mathrm{DM}$ patients and demographic data, smoking habit, denture-wearing status, $\mathrm{HbA}_{1 \mathrm{c}}$ or FPG.

\section{Haemolysin}

The $\mathrm{H}_{\mathrm{z}}$ values ranged from 0.60 to 0.79 for the DM group and from 0.62 to 0.87 for controls. Higher haemolysin activities were detected in the test group isolates $(0.673 \pm$ $0.06)$ than in the control isolates $(0.764 \pm 0.08)$ (Table 1$)$. C. albicans isolates from female type $2 \mathrm{DM}$ patients had a significantly higher haemolysin activity $(0.692 \pm 0.050)$ than their male counterparts $(0.749 \pm 0.081)$ (Student's $t$ test, $P<0.05)$. No correlation could be found between $\mathrm{H}_{\mathrm{z}}$ of isolates from type $2 \mathrm{DM}$ patients and demographic data, smoking habit, denture-wearing status, $\mathrm{HbA}_{1 \mathrm{c}}$, FPG or duration of diabetes. No significant association could be detected between $\mathrm{H}_{\mathrm{z}}$ and biotype.

Finally, no significant association was observed between phospholipase, proteinase and haemolysin activities of $C$. albicans isolates from DM or control groups.

\section{DISCUSSION}

In this study, C. albicans was isolated from $36.2 \%$ of patients with DM. This finding agrees with those from previous studies in which Candida species were isolated from between 18 and $80 \%$ of DM patients (Soysa et al., 2006). Some host factors, such as salivary flow rate, salivary $\mathrm{pH}$, wearing of dentures, alcohol use and smoking habits, are associated with an increased oral carriage rate of Candida species (Kadir et al., 2002). Virulence factors of Candida species may also be involved, although they have usually been studied in isolation and only a few researchers have investigated their role in host candidal infection/colonization (Kantarcioğlu \& Yücel, 2002; Koga-Ito et al., 2006).

Phospholipase activity was detected in $100 \%$ of the $C$. albicans isolates in this study. Previous studies have reported phospholipase activity in 30 to $100 \%$ of candidal isolates from various groups of patients and from various sites (Price et al., 1982; Wu et al., 1996). The proportion may depend on the site; for example, phospholipase activity has been found in 55, 50 and $30 \%$ of the Candida species isolated from blood, wound infections and urine, respectively (Price et al., 1982).

Phospholipase gene expression has been shown to be affected by growth conditions (Samaranayake et al., 2006). It has also been hypothesized that the presence of a high concentration of salivary glucose combined with a reduced salivary secretion rate enhances the growth of yeasts and their adherence to epithelial oral cells of type 2 DM patients (Darwazeh et al., 1991), perhaps by increasing phospholipase activity. In this study, there was no significant difference in phospholipase activity between the diabetic and non-diabetic groups, and no correlation between phospholipase activity and $\mathrm{HbA}_{1 \mathrm{c}}$ or FPG. However, we did not measure the salivary glucose concentration, which is known to be generally higher in diabetic patients (Darwazeh et al., 1991). Whether salivary glucose concentration has any effect on phospholipase gene expression warrants further investigation.

Koga-Ito et al. (2006) showed that Sap activity is significantly higher in denture wearers with signs of candidiasis than in denture wearers with a normal palatal mucosa. Another study has demonstrated that proteinase expression is not significantly higher in Candida isolates of patients with DM than in those of healthy patients, and that type 2 DM patients have higher proteinase levels than type $1 \mathrm{DM}$ patients (Manfredi et al., 2006).

The results of this study were similar to those of Koga-Ito's group: that is, there was no significant difference in oral $C$. 
albicans phospholipase activities between DM patients and controls, but proteinase activities were significantly higher in isolates from the DM group. A number of constituents in the saliva may contribute to the higher levels of oral proteinase observed in type $2 \mathrm{DM}$ patients (Manfredi et al., 2006). Higher salivary levels of glucose, IgA, and other salivary enzymes such as matrix metalloproteinase (MMP-8), gelatinase (MMP-9) and lysozyme, may all influence salivary proteinase activity and concentration in a direct or indirect fashion (Rayfield et al., 1982; Stevens et al., 1990; Collin et al., 2000). Our finding of increased in vitro proteinase activity in C. albicans isolates from DM patients, particularly those with a DM history of $\geqslant 10$ years, suggests that proteinase secretion by $C$. albicans is another plausible explanation of the increased oral proteinase activities observed in DM patients.

Studies on the activity of haemolysin in C. albicans are limited and, to the best of our knowledge, there are no studies on the activity of haemolysin from oral C. albicans isolates of type $2 \mathrm{DM}$ patients. We noted that haemolysin activity was significantly higher in oral C. albicans isolated from DM patients than in those isolated from controls.

Luo et al. (2004) studied HLP gene expression in C. albicans, but failed to obtain specific RT-PCR products. They surmise that there is either negative HLP gene expression in $C$. albicans or a relatively large amount of sequence variation in HLP genes between C. glabrata and C. albicans. Therefore, factors affecting haemolysin gene expression in C. albicans remain elusive.

Manns et al. (1994) defined the conditions under which $C$. albicans can display haemolytic activity, but found out that haemolysis is non-existent when no glucose is available in the culture medium. On the other hand, Luo et al. (2001) have tested 80 Candida isolates from clinical sources in different geographical locales and detected only alpha, and not any beta, haemolysis in experiments with glucose-free sheep blood agar. An increased blood glucose concentration may contribute, directly or indirectly, to increased haemolysin activity among C. albicans isolates in DM patients. In addition, an increased salivary glucose concentration in DM patients may also influence haemolysin production in C. albicans. Interestingly, C. albicans isolates from female DM patients showed significantly higher haemolytic activity than those from male counterparts, and female patients had higher FPG values than males. However, we were unable to detect any correlation between C. albicans $\mathrm{H}_{\mathrm{z}}$ and FPG. Further investigations are needed to clarify this issue.

A1R and J1R were the most common biotypes isolated from the test and control groups, respectively, in this study. Tsang et al. (1995) previously showed that A1R is the most common biotype isolated from HIV-infected patients in different geographical locales. Xu \& Samaranayake (1995) studied the biotypes in health and disease in China, and found that $\mathrm{A} 1 \mathrm{R}$ is only isolated in the diseased group, but that otherwise there are no significant differences. They speculate that $\mathrm{A} 1 \mathrm{R}$ is more virulent than other biotypes. Nevertheless, Samaranayake et al. (2003) found that J1R is the commonest biotype isolated from HIV-infected patients with or without oral candidiasis. This variation in biotypes regardless of their pathological source concurs with the view that $C$. albicans is an opportunist pathogen that initiates infection only when host defences are compromised (Samaranayake, 1990).

In this study, although significantly more biotype A $C$. albicans isolates were isolated from the DM group than from controls, no correlation between biotype and secretion of extracellular enzymes was noted. This finding implies that no direct relationship exists between biotype and expression of extracellular phospholipases, Saps and haemolysins. The reasons for the increased expression of Sap and haemolysin in oral C. albicans strains isolated from DM patients are as yet unknown. Possible factors, such as low $\mathrm{pH}$ and reduced salivary flow rate in DM patients, may further enhance strain selection in these patients.

Very few workers have investigated the activities of a number of extracellular enzymes in the same Candida strain. Kantarcioğlu \& Yücel (2002) found that 56 of 60 strains of C. albicans and two of four strains of Candida $k e f y r$ produce both phospholipase and protease, whereas the remaining strains do not produce either enzyme. Shimizu et al. (1996) reported that only $73 \%$ of C. albicans strains studied could produce hyaluronidase, chondroitin sulphatase, proteinase and phospholipase simultaneously; these strains were also highly virulent in mice. We noted that all C. albicans isolates from both DM and control groups expressed all three extracellular enzymes tested, and that Sap and haemolysin activities were higher in the DM group than in controls. This result suggests that the degree of extracellular enzyme activity may be important for successful oral colonization of C. albicans, at least in DM patients.

In conclusion, we report here for the first time that oral C. albicans isolates from type $2 \mathrm{DM}$ patients possess significantly higher haemolysin activity than isolates from healthy, non-DM controls. Proteinase activity of C. albicans isolates was also significantly elevated in isolates of the DM group. Our results suggest that there are both qualitative and quantitative variations in the expression of the array of extracellular enzymes secreted by C. albicans, and that they appear to be modulated by a variety of host factors pertaining to the ecosystem within which the yeast resides. Further studies on the simultaneous expression of candidal extracellular enzymes are urgently needed to understand the natural history and host-pathogen relationships associated with mucosal candidal infections.

\section{ACKNOWLEDGEMENTS}

The work described in this paper was supported by a Committee on Research and Conference Grants (CRCG) grant (10204283), The University of Hong Kong, to C.S. P. T. We thank Dr Trevor Lane for manuscript editing. 


\section{REFERENCES}

Alberti, K. G. \& Zimmet, P. Z. (1998). Definition, diagnosis and classification of diabetes mellitus and its complications. Part 1: diagnosis and classification of diabetes mellitus provisional report of a WHO consultation. Diabet Med 15, 539-553.

Belazi, M., Velegraki, A., Fleva, A., Gidarakou, I., Papanaum, L., Baka, D., Daniilidou, N. \& Karamitsos, D. (2005). Candidal overgrowth in diabetic patients: potential predisposing factors. Mycoses 48, 192-196.

Collin, H. L., Sorsa, T., Meurman, J. H., Niskanen, L., Salo, T., Ronka, H., Konttinen, Y. T., Koivisto, A. M. \& Uusitupa, M. (2000). Salivary matrix metalloproteinase (MMP-8) levels and gelatinase (MMP-9) activities in patients with type 2 diabetes mellitus. J Periodontal Res 35, 259-265.

Darwazeh, A. M., MacFarlane, T. W., McCuish, A. \& Lamey, P. J. (1991). Mixed salivary glucose levels and candidal carriage in patients with diabetes mellitus. J Oral Pathol Med 20, 280-283.

Kadir, T., Pisiriciler, R., Akyüz, S., Yarat, A., Emekli, N. \& Ipbüker, A. (2002). Mycological and cytological examination of oral candidal carriage in diabetic patients and non-diabetic control subjects: thorough analysis of local aetiologic and systemic factors. J Oral Rehabil 29, 452-457.

Kantarcioğlu, A. S. \& Yücel, A. (2002). Phospholipase and protease activities in clinical Candida isolates with reference to the sources of strains. Mycoses 45, 160-165.

Koga-Ito, C. Y., Lyon, J. P., Vidotto, V. \& Resende, M. A. (2006). Virulence factors and antifungal susceptibility of Candida albicans isolates from oral candidiasis patients and control individuals. Mycopathologia 161, 219-223.

Leung, W. K., Dassanayake, R. S., Yau, J. Y., Jin, L. J., Yam, W. C. \& Samaranayake, L. P. (2000). Oral colonization, phenotypic and genotypic profiles of Candida species in irradiated, dentate, xerostomic nasopharyngeal carcinoma survivors. J Clin Microbiol 38, 2219-2226.

Luo, G., Samaranayake, L. P. \& Yau, J. Y. Y. (2001). Candida species exhibit differential in vitro hemolytic activities. J Clin Microbiol 39, 2971-2974.

Luo, G., Samaranayake, L. P., Cheung, B. P. K. \& Tang, G. (2004). Reverse transcriptase polymerase chain reaction (RT-PCR) detection of HLP gene expression in Candida glabrata and its possible role in in vitro haemolysin production. APMIS 112, 283-290.

Manfredi, M., McCullough, M. J., Al-Karaawi, Z. M., Vescovi, P. \& Porter, S. R. (2006). In vitro evaluation of virulence attributes of Candida spp. isolated from patients affected by diabetes mellitus. Oral Microbiol Immunol 21, 183-189.

Manns, J. M., Mosser, D. M. \& Buckley, H. R. (1994). Production of a hemolytic factor by Candida albicans. Infect Immun 62, 5154-5156.

Naglik, J. R., Rodgers, C. A., Shirlaw, P. J., Dobbie, J. L., FernandesNaglik, L. L., Greenspan, D., Agabian, N. \& Challacombe, S. J. (2003). Differential expression of Candida albicans secreted aspartyl proteinase and phospholipase $\mathrm{B}$ genes in humans correlates with active oral and vaginal infections. J Infect Dis 188, 469-479.

Odds, F. C. (1998). Candida and candidosis: a review and bibliography. London: Bailliere Tindall.
Price, M. F., Wilkinson, I. D. \& Gentry, L. O. (1982). Plate method for detection of phospholipase activity in Candida albicans. Sabouraudia 20, 7-14.

Rayfield, E. J., Ault, M. J., Keusch, G. T., Brothers, M. J., Nechemias, C. \& Smith, H. (1982). Infection and diabetes: the case for glucose control. Am J Med 72, 439-450.

Samaranayake, L. P. (1990). Host factors and oral candidosis. In Oral Candidosis, pp. 66-103. Edited by L. P. Samaranayake \& T. W. MacFarlane. London: Wright.

Samaranayake, L. P., Raeside, J. M. \& MacFarlane, T. W. (1984). Factors affecting the phospholipase activity of Candida species in vitro. Sabouraudia 22, 201-207.

Samaranayake, Y. H., Samaranayake, L. P., Yau, J. Y. Y., Dassanayake, R. S., Li, T. K. L. \& Anil, S. (2003). Phenotypic diversity of oral C. albicans isolated on single and sequential visits in an HIV-infected Chinese cohort. APMIS 111, 329-337.

Samaranayake, Y. H., Dassanayake, R. S., Cheung, B. P. K., Jayatilake, J. A., Yeung, K. W. S., Yau, J. Y. Y. \& Samaranayake, L. P. (2006). Differential phospholipase gene expression by Candida albicans in artificial media and cultured human oral epithelium. APMIS 114, 857-866.

Schaller, M., Borelli, C., Korting, H. C. \& Hube, B. (2005). Hydrolytic enzymes as virulence factors of Candida albicans. Mycoses 48, 365-377.

Shimizu, M. T., Almeida, N. Q., Fantinato, V. \& Unterkircher, C. S. (1996). Studies on hyaluronidase, chondroitin sulphatase, proteinase and phospholipase secreted by Candida species. Mycoses 39, 161-167.

Soysa, N. S., Samaranayake, L. P. \& Ellepola, A. N. B. (2006). Diabetes mellitus as a contributory factor in oral candidosis. Diabet Med 23, 455-459.

Staib, F. (1965). Serum-proteins as nitrogen source for yeastlike fungi. Sabouraudia 4, 187-193.

Stevens, D. A., Odds, F. C. \& Scherer, S. (1990). Application of DNA typing methods to Candida albicans epidemiology and correlations with phenotype. Rev Infect Dis 12, 258-266.

Tsang, C. S. P. \& Samaranayake, L. P. (2000). Oral yeasts and coliforms in HIV-infected individuals in Hong Kong. Mycoses 43, 303-308.

Tsang, P. C., Samaranayake, L. P., Philipsen, H. P., McCulloug, M., Reichart, P. A., Schmidt-Westhausen, A., Scully, C. \& Porter, S. R. (1995). Biotypes of oral Candida albicans isolates in human immunodeficiency virus-infected patients from diverse geographic locations. J Oral Pathol Med 24, 32-36.

Weinberg, E. D. (1978). Iron and infection. Microbiol Rev 42, 45-66. Williamson, M. I., Samaranayake, L. P. \& MacFarlane, T. W. (1987). A new simple method for biotyping Candida albicans. Microbios 51, 159-167.

Wu, T., Samaranayake, L. P., Cao, B. Y. \& Wang, J. (1996). In-vitro proteinase production by oral Candida albicans isolates from individuals with and without HIV infection and its attenuation by antimycotic agents. J Med Microbiol 44, 311-316.

Xu, Y. Y. \& Samaranayake, L. P. (1995). Oral Candida albicans biotypes in Chinese patients with and without oral candidosis. Arch Oral Biol 40, 577-579. 